\title{
COEXPRESSION OF EPITHELIAL-MESENCHYMAL TRANSITION TWIST2 ONCOGENE AND SCHISTOSOMAL ANTIGEN REACTIVITY MARKER IN MUSCLE-INVASIVE UROTHELIAL CARCINOMA OF URINARY BLADDER PERDICT LYMPH NODE METASTASIS AT CYSTECTOMY
}

By

\section{MOHAMED WISHAHI ${ }^{1}$, HOSSAM ELGANZORY ${ }^{1}$, AMR ELKHOLY $^{1}$, HEBA KHALIL $^{2}$, MOHAMED H. BADAWY ${ }^{1}$, AHMED MEHEINA ${ }^{1}$, MAMDOUH ROUSHDY ${ }^{1}$, KHALED ESEILY ${ }^{1}$, SAMIR ELDAHSHAN ${ }^{1}$, SHADY ANIS ${ }^{3}$, NOURA KAMEL ${ }^{4}$, AND MAHMOUD ROMEIH ${ }^{5}$}

Departments of Urology ${ }^{1}$, and Pathology ${ }^{2}$, Theodor Bilharz Research Institute, Imbaba P. O. Box 30, Giza12411, and Department of Pathology ${ }^{3}$, Faculty of Medicine, Cairo University, Department of Pathology ${ }^{4}$, National Research Centre, Dokki, Giza and Department of Biochemistry ${ }^{5}$, Theodor Bilharz Research Institute, Imbaba P. O. Box 30, Giza, Egypt ( ${ }^{\star}$ Correspondence: moh.weshahy@gmail.com)

\begin{abstract}
Bladder cancer is the seventh cancer in males worldwide, approximately $25 \%$ of patients with urothelial bladder cancer (BC) present with muscle-invasive tumours, stages T2-T4, while $75 \%$ of patients present with non-muscle invasive bladder cancer (NMIBC)T1, Ta. There is a well-established relationship between schistosomiasis and urothelial carcinoma. Epithelial-mesenchymal transition Twist 2 is responsible for tumor progression and metastasis when it is overexpressed in tumour tissue and detected by immunohistochemistry (IHC). The study included 123 patients with a urothelial carcinoma of the bladder associated with or without schistosomiasis, patient's data and paraffin embedded tumour tissues were retrieved from different hospitals and archives, inclusion criteria were patients with muscle invasive bladder cancer (MIBC) of transitional cell carcinoma, exclusion criteria were squamous cell carcinoma, adenocarcinoma, and mixed variant histology, clinicopathological characteristics were: 93 patients were stage pT2-T3, No, Mo, 20 patients with NMIBC pT1, and 10 patients had pTa. The study included pre-cystectomy imaging and pathological diagnosis of 93 patients with MIBC, post-operative pathological assessment of lymph node metastasis. IHC detection in tissue samples of Twist 2 oncogene and schistosomal antigen reactivity (SAR) marker was done for the 123 patients. The results showed that MIBC harbour high expression of Twist2 indicated strong factor for lymph node metastasis. NMIBC of Ta had low Twist 2 expression, while high grade T1 had medium expression. Schistosoma antigen reactivity was over expressed in MIBC associated with schistosomiasis, but was negative in NMIBC group and in non-schistosomal MIBC. Coexpression of Twist2 and SAR with high expression was in $73.8 \%$ patients with lymph node metastasis compared to $17.6 \%$ patients with negative expression in non-schistosomal MIBC and had negative lymph node metastasis. The results were significant $(\mathrm{P}=025)$. Clinical application indicated that detection of expression of twist 2 and schistosomal antigen reactivity marker in tissue sample of trans-urethral resection of bladder tumours prior to surgery would indicate a positive lymph node metastasis in cystectomy operation showed extended lymphadenectomy and adjuvant chemotherapy, introducing imunohistochemistry in bladder cancer stratification would be of high impact on planning proper therapeutic regimen..
\end{abstract}

Key words: Egypt, Bladder cancer, Schistosomiasis, Twist2 expression, Schistosoma antigen reactivity

\section{Introduction}

Bladder cancer is the seventh cancer in the males worldwide, when males and females the eleventh in incidence (Black et al, 1997). Age-standardised incidence rate (per 100, 000 person/year) mortality worldwide in bladder cancer was 3.2 for men and 0.9 for women (Antoni et al, 2017). Compérat et al.
(2015) reported that about $25 \%$ of patients with urothelial bladder cancer (BC) present with muscle-invasive tumours, stages T2T4, while $75 \%$ of patients present with disease confined to the mucosa (stage Ta, carcinoma in situ) or submucosa (stage T1). Bladder schistosomiasis was the second commonest parasitosis after malaria, around। 
600 million people exposed to infection with schistosomiasis in Africa, Asia, South America, and Caribbean (Alfred et al, 2017). There was an established relationship between schistosomiasis and urothelial bladder carcinoma that could progress to squamous cell carcinoma (Gouda et al, 2007). For staging of bladder cancer, tumour, node, metastases (TNM) classification the one was used and the $\mathrm{pN}$ category was closely related to prognosis (Brierly et al, 2017).

Recognition of some morphological features and subtypes of urothelial carcinoma could be an important prognostic factor for treatment; among morphological subtypes were urothelial carcinoma with partial and/ or glandular differentiation, nested variant, sarcomatoid carcinoma, and mixed urothelial and squamous cell carcinoma (Qin et al, 2017). Schistosomiasis of the bladder can lead to urothelial carcinoma and/or squamous cell carcinoma (Choi et al, 2014). Overexpression of schistosomal antigen reactivity marker (SAR) in urothelial bladder carcinoma tissues was an indicator of advanced tumour and the poor prognosis (Singh et al, 2016). Epithelial-mesenchymal transition Twist 2 was responsible for tumor progression and metastasis when it was overexpressed in tissue by immunohistochemistry (Zhao et al, 2014). New pathways were discovered in development of bladder cancer and were included in this new classification (Oliveira et al, 2016). Guidance from the International Collaboration on Cancer Reporting (ICCR) helped to clarify open questions, in conjunction with the new classification. The histological groups of urothelial carcinoma evolved. Grading remained the same, despite controversy among European urologists. Substaging of pT1 tumours was recommended for the first time, and the ICCR has made recommendations on how to report this. Furthermore, worldwide advice was published on the use of immunohistochemistry, and recommendations have been made to try to standardize handling of bladder cancer from a histopathological point of view (Compérat and Varinot, 2016). At a molecular level, bladder cancer groups were stratified, and an upcoming molecular classification permits a novel view of this malignancy. At a molecular level, bladder cancer groups were stratified, and an upcoming molecular classification permits the novel view of this malignancy (Wallerand et al, 2010).

This study is a retrospective analysis on urothelial bladder cancer with and without schistosomiasis to identify the expression Twist 2 oncogenic marker and the Schistosoma antigen reactivity marker in muscle invasive bladder cancer and correlated to lymph node metastasis, over-expression of Schistosoma antigen reactivity marker in urothelial bladder carcinoma tissues was an indicator of advanced tumour and poor prognosis

\section{Material and Methods}

Patients and paraffin-embedded tissue samples: The study included 123 patients with urothelial bladder carcinoma associated with or without schistosomiasis, data and paraffin embedded tissues were retrieved from different hospitals and archives, patients with muscle invasive bladder cancer (MIBC) of urothelial type "transitional cell carcinoma" were93 cases ,selective criteria was stage pT2 T3, No, Mo on initial diagnosis with radiological imaging by computer tomography of abdomen and thorax, no previous radiotherapy or chemotherapy, no second primary malignancy, trans-urethral resection of bladder tumour (TUR-BT) establishes the diagnosis of MIBC, imaging studies showed no visible lymph nodes involvement with metastasis. Tissues were obtained from patients who had undergone total cystectomy between $2003 \&$ 2015. None of the patients had received preoperative treatment. Lymph node dissection at time cystectomy included lymph nodes up to the level of bifurcation of common iliac artery and obturator lymph nodes. Tissue samples of cystectomy specimen and retrieved lymph nodes were examined pathologically with haematoxylin and 
eosin for cancer staging, all patients were classified according to the 1997 UICC TNM classification for the stage. The study included 20 patients with non-muscle invasive bladder cancer (NMIBC) of stage T1 and 10 patients with NMIBC of stage Ta, grading system was according to OMS 2004 for the grade (LG: low grade; HG: high grade). Immunostaining was evaluated by 2 independent pathologists to validate the diagnosis. Each sample was used after written consent was obtained from the patients at time of hospital admission. Tumour recurrence/progression was defined based on clinical, radiological, or histological diagnoses. The study was approved by the hospitals. Immunohistochemistry: Twist 2 immunostaining: Immunohistochemical staining was done on paraffin-embedded tissue, 4-micrometer-thick sections were cut, using a rotation microtome. The sections were deparaffinized in xylene and rehydrated in graded alcohols Immunohistochemistry: Twist2 immunostaining: Immunohistochemical staining was done on paraffin-embedded tissue, 4-micro-meter-thick sections were cut, using a rotation microtome. The sections were deparaffinized in xylene and rehydrated in graded alcohols and distilled water. After antigen retrieval with $0.01 \%$ EDTA $(\mathrm{pH}$ 8.0 ), endogenous peroxidase activity was blocked with $1 \%$ hydrogen peroxide in distilled water for $25 \mathrm{~min}$ followed by washing with distilled water and finally PBS $+0.1 \%$ Tween for $5 \mathrm{~min}$. Twist 2 immuno-staining was done using anti-Twist 2 concentrated antibody (Abcam, ab 57997, USA), antigen retrieval was performed in all cases by steam-heating the slides in $1 \mathrm{mmol} / \mathrm{l}$ solution (pH 9.0) for $45 \mathrm{~min}$. After blocking of endogenous biotin, staining was performed using an automated immunestainer (Ultra Benchmark, Ventana, USA), followed by detection using Ultra-view detection Kid (Ventana, USA). The sections were then counterstained with Mayer's haematoxylin, upgraded alcohols, mounted, and analysed by standard light microscopy. Schistosomal antigen reactivity marker immunostaining: immune-histochemical staining was done on paraffin-embedded tissue, which had been described in details previously (Wishahi et $a l, 2014)$.

Immunohistochemistry scoring the Twist2: The entire section was examined to find the area with maximum positivity and stained nuclei for Twist2, positively stained cytoplasm and nuclei were scored using the 40X objective in 20 fields. Immunohistochemistry evaluation was done by two independent observers. For evaluation of Twist 2 expression, each slide was scored according to percentage of positively stained cytoplasm and nuclear staining. The following ranges were used: negative score was that to cells not stained, low score was that 1 to $15 \%$ positive, medium score was to $15-40 \%$ stain, high expression score was to over $40 \%$ cytoplasmic and clear expression.

Schistosomal antigen reactivity expression marker: The entire section was examined to find the area with maximum positivity stained tissues were scored using the $40 \mathrm{X}$ in 20 fields. Immunohistochemistry evaluation was done by two independent observers. For evaluation of schistosomal antigen reactivity expression, each slide was scored according density, intensity, and index.

A- Density of schistosomal antigen expression: Percentage of positively stained cells in one microscopic field is referred to as density. The positively stained cells were recorded in five consecutive fields with the highest expression. The percentage was calculated from the mean. Degrees of density of the schistosomal antigen expression were recorded as: negative $(0 \%)$; one $+(1 \%$ $25 \%)$; two ++ (26\%-50\%); three+++ (51\%$100 \%)$. Another nomenclature was: negative, low, moderate, high density. B- Intensity of Schistosoma antigen expression: Intensity described the deepness of colour intensity in the stained cells. The intensity of staining represented the body reaction towards schistosomal antigen, evaluated relative to the normal urothelial cell expression in nor- 
mal control. Positive expression of $S$. haematobium antigen was brown in colour. Degrees in intensity of schistosomal antigen expression were recorded: negative $(0 \%)$; light intensity: one $+(1 \%-25 \%)$; moderate intensity: two $++(26 \%-50 \%)$; and strong intensity: three+++ (51\% - 100\%). Another nomenclature was negative, light, moderate, and strong intensity.

C- Index of Schistosoma antigen expression: Index referred to the sum of density and intensity of schistosomal antigen expression for each case. It was the mean of density and intensity of the positive antigen expression. The index was calculated for each case independently. Four ranges of indices were identified. 0: negative; low index: $1 \%$ 25\%; medium index: $25 \%$ - 50\%; high index: $51 \%-100 \%$.

\section{Results}

The study enrolled 123 patients with urothelial bladder carcinoma, 52 were MIBC without schistosomiasis, 42 patients were MIBC with schistosomiasis, 20 cases with NMIBC high grade T1, 10 cases NMIBC Ta. Patients with MIBC had undergone cystectomy and lymphadenectomy, tissue specimens were examined for tumour staging; patient with NMIBC had undergone TURBT, high grade T1 were treated with TURBT and full course BCG intravesical instillation and follow-up of 3 years (Tab. 1).
Immunohistochemistry was applied to preoperative TUR-BT tissue samples of all patients to detect expression of Twist 2 and Schistosoma antigen reactivity marker.

Histopathological results following cystectomy showed that MIBC non- Schistosomiasis that were of high expression of Twist2 and negative expression of Schistosomal antigen reactivity marker the lymph node positive for metastasis were $17.6 \%$. MIBC with schistosomiasis and high co-expression of Twist 2 \& schistosomal antigen reactivity marker had $73.8 \%$, lymph node positive for metastasis. The immunohistochemistry of tissue sample revealed that Twist2 expression was over expressed in MIBC with and without schistosomiasis, Ta NMIBC had low Twist 2 expression, while High grade T1 NMIBC had medium expression. Schistoso$m a$ antigen reactivity was over expressed in MIBC associated with schistosomiasis, but negative in the NMIBC and in MIBC nonscistosomal. The results showed that coexpression with high expression of Twist 2 and schistosomal antigen reactivity had high impact on tumour metastasis to lymph nodes where it was positive in $73.8 \%$ compared to $17.6 \%$ in negative expression in the nonschistosomal MIBC findings were significant $(\mathrm{P}=025)$. The representative microscopic pictures were shown (Fig. 1).

Table 1: Clinicopathological characteristics of patients with muscle-invasive bladder cancer non -Schistosoma $(\mathrm{n}=52)$, muscle-invasive bladder cancer schistosomal $(n=42)$, non-muscle invasive bladder cancer T1 $(n=20)$, Ta $(n=10)$. Expression of Twist 2 oncogene and schistosomal antigen reactivity marker.

\begin{tabular}{|c|c|c|c|c|}
\hline Features & $\begin{array}{l}\text { MIBC\#T2-T3, } \\
\text { non-Schistosoma }\end{array}$ & $\begin{array}{c}\text { MIBC T2-T3, } \\
\text { Schistosoma }\end{array}$ & $\begin{array}{l}\text { NMIBC" T1 } \\
\text {,high grade }\end{array}$ & $\begin{array}{l}\text { NMIBC Ta, } \\
\text { low grade }\end{array}$ \\
\hline Number & 51 & 42 & 20 & 10 \\
\hline Male & 46 & 38 & 16 & 7 \\
\hline Female & 5 & 4 & 4 & 3 \\
\hline \multicolumn{5}{|l|}{ Age-years } \\
\hline Range-mean & $57-74(60)$ & $45-61(58)$ & $44-73(61)$ & $42-67(65)$ \\
\hline Treatment & Cystectomy & Cystectomy & TUR-BT+ BCG & TUR-BT \\
\hline \multirow[t]{2}{*}{ P Tumor stage } & T2-3, No, M0 (n. 42/51) & T3, N0, M0 (n.11/42) & T1, N0, M0 & $\mathrm{Ta}, \mathrm{N} 0, \mathrm{M} 0$ \\
\hline & $\mathrm{T} 2-3, \mathrm{~N} 1, \mathrm{Mo}(n .9 / 51)$ & $\mathrm{T} 3, \mathrm{~N} 1, \mathrm{M} 0(n .31 / 42)$ & & \\
\hline Twist 2 expression & overexpression & Over expression & Medium expression & Low expression \\
\hline Scistosomal antigen expression & Negative & High positive & Negative & Negative \\
\hline
\end{tabular}

\section{Discussion}

The study included 123 patients with the urothelial carcinoma of the bladder with or without schistosomiasis. Overexpression of twist 2 has in impact on tumour progression and metastasis in urothelial bladder cancer (Wallerand et al, 2010). The results showed the MIBC harboured the high expression of 
Twist 2 indicating a strong factor for invasion and metastasis. High expression of the schistosomal antigen reactivity had been reported to signify higher tumour stage and poor survival in the MIBC (Wishahi et al, 2014). In present study, the presence of high expression of schistosomal antigen reactivity and overexpression of the Twist 2 led to high incidence of metastasis to lymph nodes that was $73.8 \%$. These findings denoted that schistosomiasis in bladder cancer played a role in tumour progression and its metastasis. El-Bolkainy et al. (2016) reported that in a series of 1095 Egyptian patients with carcinoma of the bladder treated by radical cystectomy, 902 cases $(82.4 \%)$ contained the schistosome eggs in the specimens, and 193 $(17.6 \%)$ were egg-negative. The different tumor parameters were compared in these sub-groups to explore any differences that could be related to the schistosomal infection. They added that the limited tendency to distant spread in schistosomal bladder cancer, despite its advanced local stage, was accounted for by the high frequency of low grade tumors rather than the limiting effect of local schistosomal tissue reactions. Immuno-histochemistry of bladder was recommended to standardize the bladder cancer from immuno-histopathological point of view. At a molecular and marker expression level bladder cancer has been stratified, and an upcoming molecular classification permits a novel view of this malignancy (Yun and Kim, 2013).

The present study showed that schistosomal antigen reactivity marker in association of Twist 2 were critical predictive markers for prognosis and metastasis, these data have the clinical implication in patients with the bladder cancer in endemic areas with schistosomiasis. Recognition of some morphological features and subtypes of the urothelial carcinoma could be an important prognostic factor that would assess in planning treatment decision; among the morphological subtypes are urothelial carcinoma with partial and/ or glandular differentiation, nested variant, sarcomatoid carcinoma (Brierly et $a l, 2017)$. The present findings showed that the bladder schistosomiasis associated with the bladder cancer should be considered as variant histology.

On the other hand, Abou-El-Naga (2018) reported that schistosomiasis is an infectious disease caused by a long lasting infection with a blood fluke of the genus Schistosoma. S. haematobium and S. mansoni are the species endemic in Egypt. The country has been plagued and seriously suffered from schistosomiasis over the past 5000years. Great strides had been done in controlling the disease since 1922. The history, epidemiology and the different control approaches were reviewed. Currently, Egypt is preparing towards schistosomiasis elimination by 2020 .

\section{Conclusion}

Undoubtedly, the schistosomiasis whether urinary and/or intestinal is one of the public health problem not only in Egypt but also in many agricultural countries worldwide. The recognition of some morphological features and subtypes of urothelial carcinoma could be an important prognostic factor that would assess in the planning treatment decision; among morphological subtypes were urothelial carcinoma with partial and/or glandular differentiation, nested variant, sarcomatoid carcinoma and the mixed urothelial as well as squamous cell carcinoma. So, the schistosomal/urothelial bladder cancer must be considered a histological variant.

Overexpression detection of the Twist2 and Schistosoma antigen reactivity marker in the TUR-BT bladder tumour tissue samples indicated a positive lymph node metastasis leading to lymphadenectomy during cystectomy operation, and adjuvant chemotherapy. Immunohistochemistry in bladder cancer stratification proved to be of high impact for the specific therapeutic regimen. Thus, schistosomiasis with bladder cancer must be considered a histological variant.

\section{References}

Abou-El-Naga, IF, 2018: Towards elimination of schistosomiasis after 5000 years of endemici- 
ty in Egypt. Acta Trop. 181:112-21

Alfred, Witjes, J, Lebret, T, Compérat, EM, et al, 2017: Updated 2016 EAU Guidelines on muscle-invasive and metastatic bladder cancer. Eur. Urol. 71, 3:462-75.

Antoni, S, Ferly, J, Soerjomataram, J, et al, 2017: Bladder cancer incidence and mortality: A global overview and recent trends. Eur. Urol. 71, 1:96-108.

Black, RJ, Bray, F, Ferly, J, et al, 1997: Cancer incidence and mortality in the European Union: cancer registry data and estimates of national incidence for 1990. Eur. J. Cancer 33, 7:1075107.

Brierly JD, Gospodarowicz, MK, Wittekind, C, 2017: TNM Classification of Malignant Tumours. UICC ISBN:978-1-119-26357-9 Wiley Blackwell.

Choi, W, Czerniak, B, Ochoa, A, 2014: Intrinsic basal and luminal subtypes of muscleinvasive bladder cancer. Nat. Rev. Urol. 11, 7: 400-10.

Compérat, E, Varinot, J, 2016: Immunochemical and molecular assessment of urothelial neoplasms and aspects of the 2016 World Health Organization classification. Histopathology 69, 5:717-26

Compérat, E, Larré, S, Roupret, M, 2015: Clin-icopathological characteristics of urothelial bladd- er cancer in patients less than 40 years old. Virch- ows Arch. 466, 5:589-94.

El-Bolkainy, MN, Mokhtar, NM, Ghoneim, M A, Hussein, MH, 2016: The impact of schistosomiasis on the pathology of bladder carcinoma. Wiley Online Library. https:// doi.org/ 10. 1002/ 10970142 (19811215) 48:12<2643::AIDCNCR 2820481216>3.0.CO;2-C

Gouda, I, Mokhtar, N, Bilal, D, et al, 2007: Bilharziasis and bladder cancer: a time trend analysis of 9843 patients. J. Egypt. Natl. Canc. Inst. 19, 2:158-62.
Oliveira, PA, Gil da Costa, RM, Nóbrega CV, Arantes-Rodrigues, R, Pinto-Leite, R, 2016: Challenges with in vitro and in vivo experimental models of urinary bladder cancer for novel drug discovery. Expert. Opin. Drug Discov. 11, 6:599-607.

Qin, C, Li Liang, E, Yong Du, Z, Yu Qiu, X, Tang, G, et al, 2017: Prognostic significance of urothelial carcinoma with divergent differentiation in upper urinary tract after the radical nephroureterectomy without metastatic diseases/ A retrospective cohort study. Medicine (Baltimore) 96, 21: e6945. Published online 2017 May 26. doi: 10.1097/ MD.0000000000006945

Singh, R, Ansari, JA, Maurya, N, 2016: Epithelial-to-mesenchymal transition and its correlation with clinicopathologic features in patients with urothelial carcinoma of the bladder. Clin. Genitourin. Cancer. Aug 9. pii: S1558-7673 (16) 30224-5.

Wallerand, H, Robert, G, Pasticier, G, et al, 2010: The epithelial-mesenchymal transitioninducing factor TWIST is an attractive target in advanced and/or metastatic bladder and prostate cancers. Urol. Oncol. 28, 5:473-9.

Wishahi, M, Zakarya, A, Hamamm, O, et al, 2014: Impact of density of schistosomal antigen expression in urinary bladder tissue on the stratification, cell type, and staging, and prognosis of carcinoma of the bladder in Egyptian patients. Infect. Agent Cancer 9:21-8.

Yun, SJ, Kim, WJ, 2013: Role of the epithelialmesenchymal transition in bladder cancer: from prognosis to therapeutic target. Korean $\mathrm{J}$. Urol. 54, 10:645-50.

Zhao, J, Dong, D, Sun, L, et al, 2014: Prognostic significance of the epithelial-to-mesenchymal transition markers e-cadherin, vimentin and Twist in bladder cancer. Int. Braz. J. Urol. 40, 2: 179-89.

\section{Explanation of Figure}

Representative immunohistochemical staining of Twist2 and Schistosoma antigen reactivity marker; A) Non-muscle invasive bladder cancer Ta, low expression of Twist2, Original magnification X200. B) Non-muscle invasive bladder cancer T1, medium expression of Twist2, Original magnification X200. C) Muscle invasive bladder cancer not associated with schistosomiasis, high expression of Twist2, Original magnification X200. D) Muscle invasive bladder cancer associated with schistosomiasis, high expression of schistosomal antigen reactivity marker, Original magnification X200. 


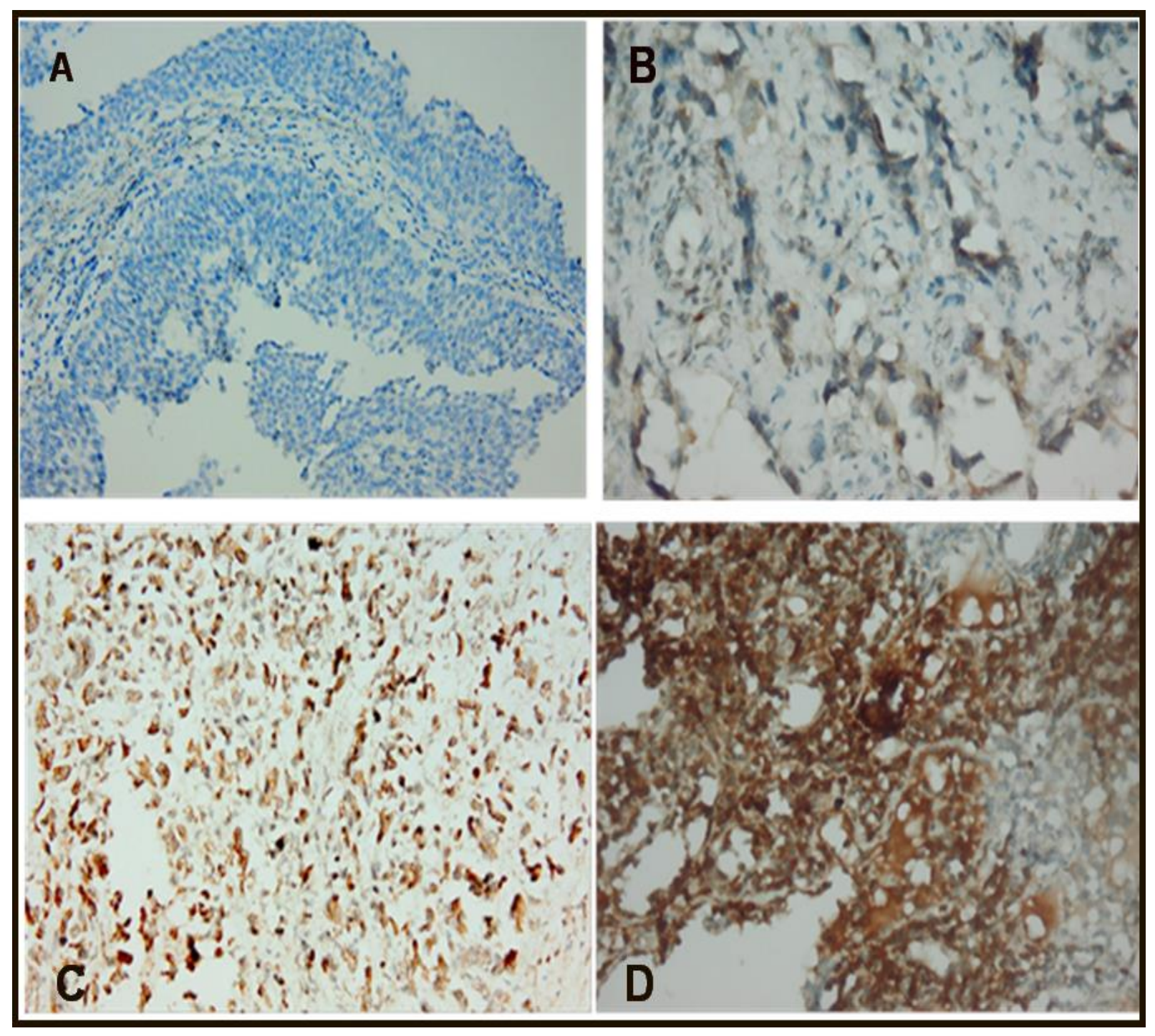

